B) Observatori de

Bioètica i Dret

ISSN: $1886-5887$

\section{Revista de Bioética y Derecho}

Publicación del Máster en Bioética y Derecho nww.bioeticayderecho.ub.edu

ARTíCULO

\title{
Il caso Italia: medicina riproduttiva e obiezione di coscienza
}

\section{The Italian case: reproductive medicine and conscientious objection}

Angela Balzano*

* Angela Balzano. Phd Student in Bioethics, Law and New Technologies at the Cirsfid - Alma Mater Studiorum, Bologne. Angela.balzano@unibo.it 


\section{Indice}

1. Legge 194 e obiezione di coscienza: la normativa italiana tra i Trattati internazionali ed europei sui Diritti Umani.

2. L'obiezione di coscienza dagli ospedali al CNB: conseguenze e limiti dei vuoti normativi e culturali.

\section{Index}

1. Law 194 and conscientious objection: the Italian legislation between the International treaties and the European Human Rights.

2. Conscientious objection from hospitals to the CNB: consequences and limits of regulatory and cultural gaps.

\section{Astratti}

Oggetto di quest'articolo sono le normative italiane, nel contesto europeo, che disciplinano l'interruzione volontaria di gravidanza, nonché l'obiezione di coscienza ad essa da parte del personale medico-sanitario. La metodologia è quella elaborata dalla Jasanoff in Fabbriche della Natura.

Dopo aver descritto la politica storica, culturale e nazionale, l'articolo prosegue con l'analisi dell'art. 9 della legge 22 maggio $1978 \backslash 194$, che regola l'obiezione di coscienza da parte del personale medico per l'interruzione volontaria di gravidanza.

La questione dell'obiezione di coscienza è inquadrata anche nel contesto europeo. A questo proposito, l'analisi si concentra sul documento che la Salute Sociale e la Commissione Affari Sociali, Salute e Famiglia presentate all'Assemblea parlamentare europea, dal titolo "Accesso delle donne a cure mediche legali: il problema di un uso non regolamentato dell'obiezione di coscienza".

In questo documento, I'Italia è vista come un caso emblematico della problematica istituzione legale dell'obiezione di coscienza, come confermato dalla Tabella 28 del Rapporto 2012 del Ministero della Salute per l'attuazione della legge 194, secondo il quale la percentuale di opposizione supera $69 \%$.

C'è una grande attenzione per il confronto tra i livelli di coinvolgimento e di azione delle diverse entità politiche, istituzionali, sociali e culturali: il ruolo dello Stato italiano nella produzione di norme e conoscenze in termini di medicina riproduttiva umana, così come il ruolo di comitati etici e delle comunità epistemiche nazionali. L'analisi si concentra sullo sviluppo del diritto europeo e internazionale, sui contributi delle categorie di professionisti, sulle opinioni di esperti di bioetica sulla questione dell'obiezione di coscienza di tecniche mediche rilevanti per la bioetica e la filosofia morale, 
come ad esempio l'interruzione della gravidanza. A tal fine, l'articolo analizza i diversi punti di vista espressi dalle associazioni professionali, come la Lega dei ginecologi non-obiettori chiamati LAIGA, e comitati di bioetica, come il Comitato Nazionale per la Bioetica, che, nel documento del 30 luglio 2012, dal titolo "Obiezione di coscienza e di bioetica", ha dichiarato di essere favorevole.

Parole chiave: biotecnologie; diritti riproduttivi; interruzione di gravidanza; obiezione di coscienza.

\section{Abstract}

The subject of this article is the Italian law in European context, regulating abortion and the conscientious objection by medical staff. The methodology is one developed by Jasanoff in Designs of Nature.

After describing the historical, cultural and national policy, the article proceeds with the analysis of art. 9 of Law May 22, $1978 \backslash 194$, which regulates conscientious objection on the part of medical personnel to the voluntary interruption of pregnancy.

The issue of conscientious objection is framed also in the European context. In this regard, the analysis focuses on the document that the Social Health and Family Issues Committee presented to the European Parliamentary Assembly, entitled "Women's access to lawful medical care: the problem of unregulated use of conscientious objection."

In this document, Italy is seen as an emblematic case of the problematic legal institution of conscientious objection, as confirmed by Table 28 of the Report 2012 of the Ministry of Health on the implementation of Law 194, according to which the percentage of objection exceeds $69 \%$.

There is a big focus on the comparison between the levels of involvement and action of different political, institutional, social and cultural entities: the role of the Italian State in producing standards and knowledge in terms of human reproductive medicine, as well as the role of ethics committees and epistemic national communities. The analysis focuses on the development of European and international law, on the contributions of the categories of professionals, on the opinions of bioethicists about the issue of conscientious objection to medical techniques relevant for bioethics and moral philosophy, such as the interruption of pregnancy. For this purpose, the article analysed the different perspectives expressed by professional associations, such as the League of not-objector gynecologists called LAIGA, and the committee of bioethicists, such as the National Bioethics Committee, which, in the document of 30 July 2012, entitled "Conscientious Objection and bioethics" declared to be favour of it.

Key words: biotechnology; reproductive rights; abortion; conscientious objection. 


\section{Legge 194 e obiezione di coscienza: la normativa italiana tra i Trattati internazionali ed europei sui Diritti Umani}

Ricostruire l'iter politico e normativo che ha portato all'approvazione della legge 194 in Italia, vuol dire riconoscere il carattere cattolico della cultura dominante. Seguendo la metodologia della Jasanoff in Fabbriche della Natura, ovvero l'analisi comparata delle culture nazionali, non possiamo non riconoscere che: la cultura politica di una nazione è rilevante nel dar forma alla politica della scienza e della tecnologia ${ }^{1} »$.

II dibattito in medicina riproduttiva è animato, in Italia, dalla dialettica tra laici e cattolici. II tema del corpo, dei suoi diritti e delle sue libertà, ha rappresentato il terreno di una battaglia culturale, in molti casi anche politica. La capacità delle donne di procreare è stata al centro di numerose polemiche mediatiche, così come è stata oggetto di encicliche papali e relazioni ministeriali. II contesto culturale italiano è segnato dall'ingerenza della morale cattolica. In questa situazione i corpi delle donne, con tutte le potenzialità di riprodurre o meno la vita, hanno a lungo svolto funzione di simbolo e di segno. II percorso che ha portato al varo della legge 194 non è stato, dunque, affatto lineare. Essa si presenta come il risultato del lavoro sinergico di più soggettività, prime tra tutte le donne. Grazie alla spinta propulsiva dei movimenti femministi dagli inizi degli anni 70 prende il via una collaborazione tra collettivi di donne, intellettuali, medici professionisti, esponenti della magistratura, parlamentari e giornalisti, al fine di scardinare le norme obsolete, risalenti al Codice penale fascista, che ancora regolavano la vita quotidiana degli individui. Come argomenta Paolo Veronesi nel suo contributo, dal titolo "Lo Statuto del corpo", pubblicato nel Trattato di Biodiritto: "Sono stati i vari movimenti per il riconoscimento dei civil rights a imporre un dibattito serrato sulla liberazione del corpo. In tal senso si pensi alle pressioni esercitate dal femminismo e dalle lotte per la libertà sessuale; alla riflessione sui temi dell'aborto e dell'identità di genere[...].Da un lato esse hanno infatti scavato nell'opinione pubblica, trasformando la coscienza e gli stessi comportamenti sociali. Gli operatori giuridici sono stati così indotti a rivolgersi alla Carta nell'intento di reperire il materiale normativo utile a risolvere i nuovi problemi $^{2}$ ». Una sentenza della Corte Costituzionale molto importante a questo proposito è la n. 27 del 18 febbraio 1975. Oggetto della sentenza è uno di quegli obsoleti articoli del codice penale criticati dai movimenti degli anni settanta: l'art. 546 del Codice Penale, che punisce chi porta a termine un' interruzione di gravidanza anche su donna consapevole ed esposta a seri rischi di salute e integrità psichica. Secondo i giudici questo articolo contrasta con il secondo comma dell'art. 31 e con il primo comma dell'art. 32 della nostra Costituzione, che ricordiamo tutelare il primo maternità e infanzia, il secondo salute individuale e collettiva. Con una felice espressione i giudici dichiarano sin dall'incipit che l'art. 546 del Codice Penale rappresenta un ostacolo per il diritto alla salute delle donne, pertanto «la dichiarazione d'illegittimità della norma consentirebbe invece a moltissime donne di poter ricorrere

1 Jasanoff, S., Fabbriche della natura. Biotecnologie e democrazia, Milano, Saggiatore, 2008, pag 36.

2 P. Veronesi, "Lo Statuto del Corpo", in Rodotà S., Zatti P., Trattato di biodiritto, , Milano, Giuffrè Editore, 2011, pag. 139. 
all'opera dei sanitari, anziché a quella pericolosissima delle fattucchiere ${ }^{3}$ ». La sentenza evidenzia, inoltre, un' altra contraddizione inerente all'allora in vigore normativa sull'aborto, quella relativa al conflitto tra i diritti del concepito e i diritti della donna. La Corte ammetteva che la tutela del concepito gode della protezione costituzionale, quella prevista dall'art. 31. Tuttavia, secondo i giudici, l'aborto non poteva più essere inquadrato nell'ambito dei "reati contro la stirpe" e punito in nome del "benessere demografico", come durante il regime fascista. Occorreva inserirlo in un ambito più confacente, quale quello dei reati contro la persona. Bisognava constatare che persona è la donna, e che ella gode dei più ampi diritti costituzionali, mentre il concepito è definibile persona solo in potenza. A questo proposito la Corte Costituzionale ha anticipato un dibattito ancora oggi molto accesso, quello intorno alla possibile definizione di persona del feto e dell'embrione. La soluzione prevista dalla Corte per questo dilemma della bioetica, è molto ancorata alla realtà, a favore del diritto delle donne alla libertà procreativa; così, infatti, si esprimono i giudici: «Non esiste equivalenza fra il diritto non solo alla vita ma anche alla salute proprio di chi é già persona, come la madre, e la salvaguardia dell'embrione che persona deve ancora diventare ${ }^{4}$ ». Questa sentenza costituisce un precedente giurisprudenziale, non prevedendo sanzioni in caso di aborto terapeutico, ed anticipa l'approvazione del testo di legge 22 maggio 1978 n. 194, intitolata "Norme per la tutela sociale della maternità e sull'interruzione volontaria della gravidanza". Questa legge permette di garantire due diritti fondamentali: il diritto alla salute e il diritto all'autodeterminazione. Prima del 1978, infatti, in Italia si eseguivano moltissimi aborti clandestini. La legge 194 trova una delle sue ragioni di essere proprio nella necessità di limitare il ricorso all'aborto clandestino, molto pericoloso per la salute della donna. La legge, tuttavia, presenta numerose imperfezioni che hanno dato luogo a spiacevoli conseguenze, una per tutte l'obiezione di coscienza da parte dei ginecologi. Vediamo, più da vicino, dunque, il testo stesso della 194. II primo articolo di questa legge stabilisce che: «Lo Stato garantisce il diritto alla procreazione cosciente e responsabile, riconosce il valore sociale della maternità e tutela la vita umana dal suo inizio. L'interruzione volontaria della gravidanza, di cui alla presente legge, non e' mezzo per il controllo delle nascite. Lo Stato, le regioni e gli enti locali, nell'ambito delle proprie funzioni e competenze, promuovono e sviluppano i servizi socio-sanitari, nonché altre iniziative necessarie per evitare che lo aborto sia usato ai fini della limitazione delle nascite ${ }^{5}$. Con l'art. $4 \mathrm{si}$ entra nel vivo della questione, e compare finalmente il diritto delle donne all'interruzione volontaria di gravidanza. Essa è permessa entro i primi 90 giorni e può essere richiesta per svariati motivi. II legislatore in quest'art. specifica che essa può essere ottenuta quando è a rischio la salute fisica e psichica della donna, quando le difficoltà economiche, sociali e familiari costituiscono un impedimento alla buona riuscita della gravidanza, quando la situazione in cui è avvenuto il concepimento è critica (in caso di stupro), quando sono accertate gravi malformazioni o patologie del feto. In tutti questi casi le donne italiane hanno il diritto di rivolgersi a un consultorio pubblico e ottenere il necessario certificato. All'art. 5 si legge che i consultori, prima di rilasciare tale consenso, devono assicurarsi che 
le cause che inducono la donna ad abortire non siano irrimediabili. Nel caso in cui, invece, il medico della struttura in questione, riconosce fondatezza e urgenza, egli è tenuto a rilasciare «immediatamente alla donna un certificato» ${ }^{6}$.

L' articolo 9 riguarda direttamente l'obiezione di coscienza. Esso prevede la possibilità per il personale sanitario di avvalersi dell'obiezione di coscienza in materia di interruzione di gravidanza. Tale obiezione non può essere invocata per le procedure mediche antecedenti o seguenti l'intervento di aborto. Pur ammettendo l'obiezione, il legislatore sottolinea che le strutture sanitarie devono «assicurare lo espletamento delle procedure previste dall'articolo 7 e l'effettuazione degli interventi di interruzione della gravidanza richiesti secondo le modalità previste dagli articoli 5,7 e 8» e che in aggiunta «la regione ne controlla e garantisce l'attuazione anche attraverso la mobilità del personale ${ }^{7}$ ». L'obiezione di coscienza è, inoltre, oggetto di normative internazionali sui diritti umani. A questo proposito ricordiamo che il Comitato del CEDAW ha espresso la sua preoccupazione sulle difficoltà nell'accesso all'IVG causate dalle leggi che permettono l'obiezione di coscienza. II Comitato ha chiarito che è compito del governo nazionale assicurare che vi siano alternative per garantire $\mathrm{i}$ diritti riproduttivi delle donne. Secondo il Comitato, il servizio pubblico nazionale dovrebbe sempre assicurare l'accesso alle procedure di IVG, anche obbligando il personale medico a non ostacolare le stesse, in nome del diritto delle donne a non essere costrette a portare a termine la gravidanza ${ }^{8}$. Anche il Comitato per i Diritti Umani ha sollevato il problema dell'obiezione di coscienza del personale medico alle pratiche di IVG, sottolineando soprattutto la mancanza di informazioni e dati a riguardo, e sollecitando gli stati a monitornarne diffusione e conseguenze ${ }^{9}$. In ambito europeo è stata, poi, avanzata una proposta per regolamentare in maniera più omogenea la pratica dell'obiezione di coscienza nel contesto della salute riproduttiva. II 20 luglio 2010 il Comitato Questioni Sociali Salute e Famiglia porta all' attenzione dell' Assemblea Parlamentare Europea un documento intitolato Women's access to lawful medical care: the problem of unregulated use of conscientious objection, presentato da Ms Christine McCafferty. ${ }^{10}$ Pur riconoscendo il diritto all'obiezione di coscienza nell'esecuzione di alcune pratiche mediche, il Comitato si dice molto preoccupato riguardo alla diffusione, in alcuni paesi europei, spropositata e non regolamentata, di questa stessa, nell'ambito della salute riproduttiva. II Comitato sottolinea che vi è la necessità di bilanciare il diritto all'obiezione di coscienza con il principio della responsabilità professionale e con il diritto dei pazienti di accedere alle cure mediche in tempi adeguati. L'assemblea parlamentare dovrebbe invitare i paesi membri ad elaborare una regolamentazione complessiva al fine di normare l'obiezione di coscienza nei riguardi delle cure mediche, incluse le cure per la salute riproduttiva, di monitorarla costantemente e di tenerla entro certi parametri. Al secondo punto delle raccomandazioni suggerite da questo Comitato,

6 Art. 5, Legge 22 maggio 1978 n 194.

7 Art. 9, Legge 22 maggio 1978 n 194.

8 Italia, 353, U.N Doc. A/52/38 Rev.1, Parte II, 1997; Polonia,25 U.N Doc. CEDAW/C/POL/CO/6, 2007.

9 Polonia, 8, U.N DOC CCPR/CO/82/POL, 2004.

10 Women's access to lawful medical care: the problem of unregulated use of conscientious objection, ,presentato da Ms Christine McCafferty, United Kingdom, Socialist Group, 20\07\2010. 
l'obiezione di coscienza è definita esplicitamente come «un ostacolo per le donne nell'accesso alle cure mediche garantite dalla legge ${ }^{11}$ ». I trattati internazionali ed europei sui diritti umani riconoscono il diritto alla libertà religiosa e di coscienza, ma tutelano al contempo il diritto dei cittadini di accedere ad adeguate cure mediche, comprese quelle legate alla salute riproduttiva. Quando questi due diritti entrano in conflitto, gli stati hanno l'obbligo di assicurare i servizi medici previsti dall'ordinamento così come di fornire ogni prodotto farmacologico, in modo da «non arrecare svantaggi o danni ai pazienti legalmente titolari dei diritti alla salute ${ }^{12}$. L'art. 9 della Convenzione Europea sui diritti umani garantisce il diritto alla libertà di pensiero e di coscienza ma afferma che questo diritto è soggetto alle limitazioni previste dalla legge, necessarie in una società democratica per l' interesse della sicurezza e della salute pubblica, per la tutela della libertà degli individui. Questa limitazione è stata esplicitamente riconosciuta dalla Corte Europea sui Diritti Umani per quanto riguarda il libero accesso alla contraccezione ${ }^{13}$. II Comitato fa, poi, riferimento a codici professionali internazionali, come I' OMSA e la FIGO ${ }^{14}$. Entrambi questi organi affermano che i medici obiettori sono tenuti ad informare immediatamente della loro obiezione ed offrire valide ed istantanee alternative in grado di esaudire le richieste. Nel caso di impossibilità per la paziente di ricorrere ad alternative, o in caso di rischio di vita e salute, i medici obiettori sono tenuti a prestare le cure mediche necessarie, e non hanno possibilità di appellarsi alla libertà di coscienza. Entrambi chiariscono che l'obiezione di coscienza riguarda i singoli professionisti, non le strutture, che, poiché pubbliche, sono obbligate dalla legge a prestare in ogni momento i servizi sanitari previsti dall'ordinamento, e non possono danneggiare salute e vita delle donne rifiutandosi di eseguire trattamenti previsti dalla legge ${ }^{15}$. II Comitato riconosce, poi, che non tutti i paesi membri godono della stessa normativa. Per questa disparità nella regolamentazione giuridica il Comitato auspica che sia fatta omogeneità almeno per quanto riguarda i doveri degli obiettori, affermando che «gli Stati dovrebbero certificare che le norme sull'obiezione di coscienza specifichino i doveri ad essa connessi. L'assenza di precisi limiti legali e istituzionali in molti paesi si traduce nell'impossibilità per i cittadini di accedere alle cure mediche previste dall'ordinamento, minando in questo modo il lorodiritto alla salute e alla privacy, e costituisce un'infrazione del dovere di cura nei confronti del paziente nonchè una negligenza medica espressa con l'abbandono dello stesso ${ }^{16}$ ". Alcuni paesi, invece, presentano normative specifiche sull'obiezione di coscienza del personale medico all'IVG, come Norvegia, Slovenia, Gran Bretagna: le linee guida del Servizio di Salute Nazionale del Regno Unito definiscono dovere medico quello di interrompere una gravidanza laddove richiesto dalla donna, e prevedono una selezione adeguata del personale sanitario,

11 Ivi.

12 Report of the United Nations CEDAW Committee, 2008.

13Pichon and Sajous v. France, European Court of Human Rights, Application No. 49853/99 (admissibility decision), and Adriana Lamacková, Conscientious Objection in Reproductive Healthcare: Analysis of Pichon and Sajous v. France, European Journal of Health Law 15 (2008).

14 Federazione internazionale di ginecologici e ostetriche.

15 WHO, Safe Abortion Guidance (2003). 15 FIGO: Resolution on Conscientious Objection, 2006.

16 Women's access to lawful medical care: the problem of unregulated use of conscientious objection, presentato da Ms Christine McCafferty, United Kingdom, Socialist Group, 20\07\2010. 
esplicitando che i ginecologi devono essere pronti a «soddisfare tutti i doveri a cui sono chiamati a rispondere, inclusi i doveri connessi all'interruzione di gravidanza ${ }^{17}$ ».

Secondo il Comitato è la mancanza di organi di monitoraggio e controllo dell'obiezione di coscienza a costituire una delle cause della discriminazione in atto sulle donne nell'accesso alle cure mediche riproduttive. A questo proposito il Comitato cita la relazione del Ministero della Salute italiano del 2008, perché ritiene che tale assenza abbia causato, in Italia, una sproporzione tra medici obiettori e medici non obiettori. La relazione ministeriale riporta che quasi il 70\% dei ginecologi italiani si rifiuta di praticare l' aborto per ragioni morali, nonostante la presenza di una legge che lo legalizza completamente $^{18}$. Nelle conclusioni il Comitato invita, pertanto, gli Stati ad elaborare una precisa e chiara normativa che bilanci il diritto alla libertà di coscienza del personale medico con quello alle cure, garantite dalla legge, dei pazienti. Inoltre, il Comitato invita i paesi ad istituire meccanismi di monitoraggio e un registro del personale sanitario che esercita l'obiezione. I doveri previsti dal Comitato per il personale obiettore consistono nell'obbligo di: informare i pazienti su tutte le scelte disponibili e sulla propria obiezione di coscienza in tempi adeguati; informare sulle alternative per soddisfare l'esigenza di cure dei pazienti; non sottrarsi alla cura medica richiesta nei casi di inesistenza di una valida alternativa, o in casi di emergenza. In chiusura, il Comitato afferma che "tutte le normative nazionali dovrebbero prevedere l'istituzione di un efficace organo di controllo sugli abusi nella pratica dell'obiezione di coscienza, che garantisca alle donne rimedi sicuri ed efficienti ${ }^{19,}$.

\section{Donne bloccate tra scienza e diritto: L'obiezione di coscienza dagli ospedali al CNB: conseguenze e limiti dei vuoti normativi e culturali}

Seguendo la distinzione della Jasanoff, asseriamo che nostro intento è mostrare come bioetica ufficiale e bioetica non ufficiale influiscano sulle scelte normative e mediche nazionali, e quali siano le conseguenze sulle soggettività interessate, ovvero sulle donne che richiedono l'IVG. Seguiremo l'attività del ministero della Salute e quella del $\mathrm{CNB}^{20}$, riferendoci ad essi come istituti di governo e di bioetica ufficiale, di «bioetica intesa come strumento di policy pubblica ${ }^{21}$ ». Analizzeremo, poi, l'attività di Ass. di categoria come la $L A / G A^{22}$ e del Comitato di Bioetica Laica Nazionale, attori della bioetica

17 United Kingdom, NHS Guidelines, Appointment of doctors to hospital posts, NHS Executive HSG(94)39, 14 September 1994.

18 Republic of Italy, Ministry of Health, Report of the Ministry of Health on the Performance of the Law Containing Rules for the Social Care of Maternity and Voluntary Interruption of Pregnancy: 2007-2008.

19 Women's access to lawful medical care: the problem of unregulated use of conscientious objection", presentato da Ms Christine McCafferty, United Kingdom, Socialist Group, 20\07\2010.

20 Comitato Nazionale di Bioetica.

21 Jasanoff, S., Fabbriche della natura. Biotecnologie e democrazia, Milano, Saggiatore, 2008, pag. 209.

2223 www.laiga.it, Libera Associazione Italiana dei Ginecologi per l'applicazione della legge 194/78. 
non ufficiale, dell'etica pubblica intesa come discorso strategico delle soggettività non istituzionali, che prendono parola per raggiungere i propri fini normativi in fatto di medicina riproduttiva.

II Ministro della Salute Renato Balduzzi ha presentato in data 8 ottobre 2012 la relazione sull'attuazione della legge 194\1978. Al punto 3.10 di questo report si leggono i dati più attuali su diffusione ed esercizio dell'obiezione di coscienza all'IVG. La tabella 28 di tale relazione mostra le percentuali di obiezione per categorie professionali. In Italia la percentuale di ginecologi obiettori è passata dal $58.7 \%$ del 2005 , al $69.2 \%$ del 2006 , al $70.5 \%$ del 2007 , al $71.5 \%$ del 2008 , al $70.7 \%$ nel 2009 e al 69.3 nel 2010; per gli anestesisti dal 45.7\% al 50.8\%. Per il personale non medico si è osservato un ulteriore incremento, con percentuali che dal 38.6\% nel 2005 sono arrivate al $44.7 \%$ nel 2010. Percentuali superiori all" $80 \%$ tra i ginecologi si osservano soprattutto al sud: $85.7 \%$ in Molise, 85.2\% in Basilicata, 83.9\% in Campania, $81.3 \%$ a Bolzano e $80.6 \%$ in Sicilia. Anche per gli anestesisti i valori più elevati si osservano al sud (con un massimo di più di $75 \%$ in Molise e Campania e $78.1 \%$ in Sicilia) e i più bassi in Toscana (27.7\%) e in Valle d"Aosta (26.3\%). Per il personale non medico i valori sono più bassi e presentano una maggiore variabilità, con un massimo di $86.9 \%$ in Sicilia e 79.4\% in Calabria. Queste statistiche si traducono in una realtà di negazione di diritti e di libertà, nella misura in cui è sempre più difficile per una donna accedere all'interruzione volontaria di gravidanza. In molti ospedali italiani vi è totale assenza di personale non obiettore, e così le donne sono costrette a mettersi alla ricerca di una struttura vicina in grado di offrire il servizio richiesto. Questo determina un rallentamento dei tempi di questa operazione, e mina il diritto delle donne alla salute e all'autodeterminazione. Un altro diritto che l'obiezione di coscienza lede è il diritto delle donne a non essere discriminate in base al sesso: essa assomiglia sempre di più a una discriminazione di genere, nella misura in cui l'interruzione volontaria di gravidanza è un intervento medico che riguarda solo le donne, e che proprio ad esse viene negato. Vi è un secondo livello di discriminazione tra donne stesse, che viaggia su linee di classe: una donna facoltosa, dotata di mezzi economici e culturali, può permettersi di spostarsi verso l'ospedale con personale non obiettore, addirittura può ricorrere a cliniche private per l'IVG, al contrario di una meno abbiente. La legge 194, all' art. 9 concede al personale medico-sanitario di esercitare obiezione di coscienza sull'IVG, senza regolamentarne il ricorso. II legislatore non ha fissato alcun tetto, alcun parametro. Si limita a dire che è competenza delle regioni monitorare sulla corretta proporzione tra obiettori e non obiettori. L'unico paletto posto riguarda l'attività privata del medico obiettore: egli non può eseguire IVG nelle strutture private se si è dichiarato obiettore in un ente pubblico. Nei casi in cui si riscontri tale contraddizione, l'obiezione si intende revocata: «L'obiezione di coscienza si intende revocata, con effetto immediato, se chi l'ha sollevata prende parte a procedure o a interventi per l'interruzione della gravidanza previsti dalla presente legge, al di fuori dei casi di cui al comma precedente ${ }^{23}$ ». L'art 9 della 194 ammette l'obiezione senza chiedere nulla in cambio, per cui medici e personale sanitario possono rifiutarsi di eseguire aborti senza dover prestare alcun servizio supplementare. La situazione si presenta come illogica: aumentano gli obiettori di coscienza e diminuisce la possibilità di ricorrere all'IVG, la stessa 
libertà procreativa. La LAIGA, in un rapporto stilato insieme alla $\mathrm{IPPF}^{24}$, annuncia che tra poco più di 5 anni in Italia la 194 verrà completamente disapplicata a causa del dilagare dell'obiezione di coscienza. Per questo motivo quest'associazione, nel novembre 2012, ha scritto una lettera al ministro della Salute, chiedendogli di cercare una soluzione a proposito. Qui, la LAIGA fa notare come l'assenza di monitoraggio del fenomeno sia parte del problema, scrivendo: «Abbiamo recentemente svolto un monitoraggio dello stato di applicazione della legge 194 nel Lazio, che ci ha confermato la sensazione dell'esistenza di uno scollamento fra i dati ufficiali contenuti nella relazione al Parlamento del precedente Ministro della Salute, e i dati reali da noi raccolti. ${ }^{25}$ » Inoltre, la LAIGA ha portato il caso Italia all'attenzione del Consiglio d'Europa, nella fattispecie del suo "Comitato Europeo per i diritti sociali": un vero e proprio ricorso, presentato insieme a IPPF, contro lo Stato italiano, accusato di non garantire il diritto alla salute e all'autonomia delle donne. Dal loro comunicato si legge: «Nel regolare l'obiezione di coscienza degli operatori sanitari, l'art. 9 non indica le misure concrete che gli ospedali e le regioni devono attuare per garantire un'adeguata presenza di personale non obiettore in tutte le strutture sanitarie pubbliche, in modo da assicurare l'accesso alla procedure per l'interruzione di gravidanza. II numero insufficiente di medici non obiettori mina, il diritto delle donne alla salute e discrimina quelle che per motivi finanziari non possono recarsi in un'altra regione 0 in strutture private ${ }^{26}$ ». In ambito italiano, inoltre, si batte contro l'obiezione di coscienza il Comitato di bioetica laico nazionale, promotore della campagna II buon medico non obietta. Suo obiettivo è l'abrogazione completa dell'art. 9, ovvero il divieto di appellarsi all'obiezione per i ginecologi di fronte a una richiesta di IVG. Dal loro Manifesto contro l'obiezione di coscienza, del giugno 2012, si evince una visione liberal-democratica della società, in cui non vi è spazio per l'obiezione perché questa limita il diritto delle donne a vivere secondo le proprie scelte e i propri valori. Per questo il Comitato di bioetica laica afferma che: «Chi nega il diritto all'obiezione di coscienza in sanità non intende negare il valore dell'autonomia personale ma è impegnato nella difesa dei diritti civili fondamentali ${ }^{27}$ ». Ad un primo impatto la soluzione proposta dal Comitato di bioetica laica pare radicale: I' abrogazione completa dell'art. 9 non si presenta senz'altro come una mediazione. Essa, come scrive il costituzionalista Veronesi, è piuttosto un extrema ratio, motivata dal fatto che l'obiezione di coscienza aveva un senso «nel momento in cui entrò in vigore la legge 194, ma non avrebbe più alcuna ragion d'essere per coloro che oggi optano per cimentarsi con una determinata specializzazione medica, ben sapendo cosa li attende (questa posizione è stata sostenuta in varie sedi, e tra gli altri, da Stefano Rodotà28)». Questa proposta è dettata dal dilagare del fenomeno stesso dell'obiezione, che, viste le percentuali, mette in seria discussione l'attuazione reale e uniforme su tutto il territorio nazionale del diritto

24 www.ippf.org, The International Planned Parenthood Federation

25 http://www.laiga.it/index.php?option=com content\&view=article\&id=128:lettera-aperta-al-ministro-

della-salute-renato-balduzzi-e-pc-alla-ministra-del-lavoro-e-delle-pari-opportunita-elsa-fornero-

\&catid $=42:$ notizie $\&$ Itemid $=93$

26 Comunicato stampa 23 novembre 2012, Laiga-ippf, fonte ANSA.

27 http://obiettoridicoscienzano.wordpress.com/about/

28 S.Canestrari, F. Mantovani, P. Veronesi, L'obiezione di coscienza e le riflessioni del giurista nell'era del biodiritto, pag 411. Criminalia, Vol. n. 1, Edizioni ETS, Pisa, 2011. 
all'aborto. Attestata la situazione di estrema precarietà in cui versano diritto alla salute e all'autodeterminazione delle donne italiane causa il diffondersi dell'obiezione di coscienza, guardiamo alla posizione del CNB, principale organo consultivo in quest'ambito. II CNB, nel documento del 30 luglio 2012 intitolato Obiezione di Coscienza e Bioetica, si propone di analizzare gli aspetti morali e giuridici della stessa, definita come diritto della persona e istituzione democratica, capace di garantire il pluralismo delle idee. II CNB afferma che «l'obiezione di coscienza in bioetica è costituzionalmente fondata [...]; essa costituisce un diritto della persona e un'istituzione democratica necessaria a tenere vivo il senso della problematicità riguardo ai limiti della tutela dei diritti inviolabili; quando l'obiezione di coscienza inerisce a un'attività professionale, concorre ad impedire una definizione autoritaria ex lege delle finalità proprie della stessa attività professionale ${ }^{29}$. II ragionamento del CNB può essere così riassunto: l'obiezione di coscienza è legittima in campo bioetico quando riguarda problematiche connesse ai diritti umani inviolabili (il diritto alla vita); uno stato democratico non può obbligare dei professionisti alla mera esecuzione dei servizi garantiti dalla legge; l'obiezione risulta compatibile con la legalità perché non comporta la disobbedienza alle leggi statali, ma solo la sottrazione ad esse da parte di alcuni professionisti in nome della libertà di coscienza; l'obiezione non deve prevedere doveri aggiuntivi poiché non è una concessione bensì un diritto fondamentale della persona. Eppure lo stesso CNB ammette che il diritto all'obiezione non può entrare in collisione con i diritti dei pazienti previsti dalle legge, e a questo scopo nelle conclusioni raccomanda «la predisposizione di un'organizzazione delle mansioni e del reclutamento, negli ambiti della bioetica in cui l'obiezione di coscienza viene esercitata, che può prevedere forme di mobilità del personale e di reclutamento differenziato atti a equilibrare, sulla base dei dati disponibili, il numero degli obiettori e dei non obiettori » ${ }^{30}$. II CNB non condanna l'aborto, dal momento che sottolinea che l'obiezione di coscienza non dovrebbe essere un modo per invalidare una legge nazionale come la 194. Tuttavia, quest'argomentazione risulta debole se confrontata con quella, molto più estesa nel documento, a favore della libertà di coscienza morale dei professionisti medici. II Professor Carlo Flamigni sottolinea questa incongruenza nella sua Postilla al documento, scritta per motivare il suo voto contrario al documento. Questa postilla ha il merito di ricordarci la pluralità delle posizioni in campo, impossibili da ridurre ad un' unica visione morale, coincidente con quella cattolica a difesa del valore della vita prenatale. Carlo Flamigni riporta le voci dei giuristi che dissentono dall'opinione del CNB, quali Garino, Capograssi e Piola. Garino ritiene che l'obiezione andrebbe contenuta e regolamentata, per renderla giuridicamente credibile, e che sarebbe necessario prevedere doveri aggiuntivi per quei professionisti che vi fanno ricorso. Inoltre, Flamigni avanza l'ipotesi che l'obiezione di coscienza sia un istituto giuridico pericoloso e contraddittorio, citando l'opera del costituzionalista Gladio Gemma. Quest'ultimo definisce l'obiezione di coscienza come un diritto legalmente codificato alla disobbedienza civile, fonte di incongruenze giuridiche e antinomie inconciliabili. Egli si chiede come può uno Stato ammettere secundum legem la possibilità di non rispettare una sua stessa legge. Le conseguenze di questa illogicità non sono certo da sottovalutare. La prima conseguenza consiste nel pericolo che «venga

29 Comitato Nazionale di Bioetica, Obiezione di Coscienza e Bioetica, 30 luglio 2012.

30 Comitato Nazionale di Bioetica, Obiezione di Coscienza e Bioetica, 30 luglio 2012. 
riconosciuta una prevalenza indiscriminata della coscienza individuale su qualsiasi precetto legale che con essa entri in collisione ${ }^{31}$. La seconda conseguenza non è meno preoccupante, in quanto potrebbe comportare l'erosione delle norme di interesse pubblico: «Si arriverebbe così a una situazione paradossale, quella di uno stato che su mandato dei cittadini indica comportamenti vincolanti e che contemporaneamente consente che delle minoranze possano rifiutarsi di prestare determinati servizi opponendosi in questo modo alla volontà popolare, in totale contrasto con la logica della democrazia. Nella fattispecie la volontà della maggioranza, che ha chiesto la legalizzazione dell'aborto, verrebbe annullata da una maggioranza di medici obiettori, una chiara sconfitta della logica oltre che della democrazia» ${ }^{32}$. Sia Gemma che Flamigni sono dell'opinione che l'obiezione di coscienza, in un ordinamento costituzionale, non può configurarsi come libertà personale. Qualora vi siano delle norme che contrastano con i principali diritti umani, esse possono essere eliminate attraverso gli strumenti di garanzia costituzionale, senza il ricorso all'obiezione del singolo. Non è possibile assimilare l'obiezione di coscienza al diritto all'autodeterminazione, essendo i due diritti diversi tra loro: «Per esemplificare, una cosa è la libertà di curarsi o meno, altro è la pretesa del medico di non curare chi ha il diritto di essere curato ${ }^{33}$ ». Accanto alle argomentazioni espresse dal costituzionalista Gemma, si collocano quelle di un altro noto costituzionalista italiano, il giurista Paolo Veronesi, che nelle sue opere parte proprio dal ribadire che «l'obiezione di coscienza non deve ledere, in concreto, il contrapposto diritto di chi chiede l'osservanza della norma rifiutata, il quale andrà comunque tutelato. Ciò costituisce il miglior antidoto attraverso il quale il legislatore può davvero garantirsi che, mediante il grimaldello della coscienza, taluni non puntino, in realtà, a perseguire obiettivi di carattere squisitamente politico ${ }^{34}$. Veronesi teme che, in materia di interruzione di gravidanza, l'obiezione di coscienza stia minando il principio di bilanciamento di diritti a favore dei medici e a svantaggio delle donne, e a questo proposito così si esprime: «può insomma concludersi che il bilanciamento tra gli interessi costituzionalmente propri delle pazienti e degli obiettori, predisposto dalla legge in base alle indicazioni della Consulta, pare ormai sul punto di saltare; stando anzi ai dati dell'ultima Relazione ministeriale sull'attuazione della legge 194, in talune zone del paese esso sarebbe già del tutto franato ${ }^{35}$ ». Bisogna riconoscere che non tutti i medici obiettori seguono $i$ dettami della propria coscienza: «La massa montate dell'obiezione all'aborto è dettata non già da precise opzioni di coscienza bensì da sempre più sospette ragioni di comodo, di carriera, di realizzazione professionale, le testimonianze sul punto sono ormai numerose e più che credibili ${ }^{36}$ ». A riguardo il CNB sostiene che, data l'impossibilità di controllare le ragioni che conducono all'obiezione, bisogna tollerare una parte di obiettori non proprio sinceri. Flamigni fa notare come in questo modo il

31 Flamigni C., Postilla al Doc. del CNB "Obiezione di coscienza e bioetica", pag.23, 2012.

32 Flamigni C., Postilla al Doc. del CNB "Obiezione di coscienza e bioetica", pag.23, 2012.

33 Gemma G., Postilla del Prof. Falmigni C. al Doc. del CNB "Obiezione di coscienza e bioetica", pag.23, 2012. 34 S.Canestrari, F. Mantovani, P. Veronesi, L'obiezione di coscienza e le riflessioni del giurista nell'era del biodiritto, pag 406. Criminalia, Edizioni Ets, Pisa, Vol. n. 1, 2011.

35 S.Canestrari, F. Mantovani, P. Veronesi, L'obiezione di coscienza e le riflessioni del giurista nell'era del biodiritto, pag 408. Criminalia, Edizioni ETS, Pisa, Vol. n. 1, 2011.

36 Ivi. 
CNB si presti a ragionamenti illogici: come si può, infatti, ammettere il diritto all'obiezione in nome delle credenze morali individuali, e poi concedere che un numero non precisato di medici farà ricorso allo stesso diritto per ragioni di tutto altro carattere? Occorre considerare che quella del ginecologo nelle strutture ospedaliere pubbliche non è una professione a cui si è costretti, ma una libera scelta dei giovani al termine degli studi. Nel caso assurdo che tutti fossimo costretti a fare i ginecologi, allora potrebbe forse valere il diritto all'obiezione. E' questa la fattispecie della leva militare, che un tempo riguardava tutti i maggiorenni di sesso maschile. Ginecologici e ostetriche, però, scelgono liberamente queste professioni in un'età in cui sono in grado di valutare le conseguenze delle loro decisioni. Sembra quantomeno illogico prevedere che essi possano rifiutarsi di eseguire dei compiti legati al loro lavoro, garantiti da una legge dello Stato, in nome della propria morale. La scelta di fare il ginecologo andrebbe accompagnata da una maggiore consapevolezza degli obblighi che questa professione comporta. Come ricorda Flamigni: «Fare il ginecologo significa impegnarsi prima di tutto a proteggere la salute della donna; interrompere una gravidanza non desiderata significa ancora la stessa cosa, proteggere la salute della donna» ${ }^{37}$.

\section{Bibliografia}

- S.Canestrari, F. Mantovani, P. Veronesi, L'obiezione di coscienza e le riflessioni del giurista nell'era del biodiritto, Criminalia, Edizioni ETS, Pisa, Vol. n. 1, 2011.

- Comitato Nazionale di Bioetica, Obiezione di Coscienza e Bioetica, 30 luglio 2012, www.governo.it.

- Flamigni C., Postilla al Doc. del CNB Obiezione di coscienza e bioetica, 2012, www.governo.it.

- Jasanoff, S., Fabbriche della natura. Biotecnologie e democrazia, Milano, Saggiatore, 2008.

- Legge 22 maggio 1978 n 194, www.governo.it.

- Relazione Parlamento IVG 2012, Ministero della Salute, www.salute.gov.it.

- Rodotà S. Zatti P., Trattato di biodiritto, Milano,Giuffrè Editore, 2011.

- Sentenza n. 27\18 febbraio 1975\ Corte Costituzionale.

- Women's access to lawful medical care: the problem of unregulated use of conscientious objection, Ms Christine McCafferty, United Kingdom, Socialist Group, 20107\2010, www.assembly.coe.int.

Fecha de recepción: 12 de marzo de 2013

Fecha de aceptación: 20 de mayo de 2013

37 Flamigni C., Postilla al Doc. del CNB "Obiezione di coscienza e bioetica", pag 22, 2012. 